\title{
Inhibition of the norepinephrine transporter to treat neurogenic orthostatic hypotension: is this the end of the story?
}

\author{
Guillaume Lamotte ${ }^{1} \cdot$ Patricio Millar Vernetti ${ }^{2}$ \\ Published online: 10 November 2021 \\ (c) The Author(s), under exclusive licence to Springer-Verlag GmbH Germany 2021
}

Keywords Autonomic $\cdot$ Multiple system atrophy $\cdot$ Ampreloxetine $\cdot$ Parkinson disease $\cdot$ Pure autonomic failure

Neurogenic orthostatic hypotension (nOH) is the most common clinical manifestation of cardiovascular autonomic dysfunction in the synucleinopathies, a group of neurodegenerative clinical syndromes including pure autonomic failure, Parkinson disease, dementia with Lewy bodies, and multiple system atrophy. The presence of $\mathrm{nOH}$ in the synucleinopathies has been associated with a more rapid disease progression, shorter survival time, higher incidence of hospitalizations, and higher risk for cognitive impairment and falls [3, 4]. Available therapeutic options for $\mathrm{nOH}$ are limited, and include sympathomimetic such as midodrine, the norepinephrine precursor droxidopa, the mineralocorticoid agonist fludrocortisone, and the reversible acetylcholinesterase inhibitor pyridostigmine [9]. Among these, only midodrine and droxidopa have been approved by the U.S. Food and Drug Administration (FDA) for the treatment of nOH. Some of the limitations of these medications include side effects (e.g., headache, scalp pruritus, diarrhea, urinary retention, supine hypertension), lack of response in a significant subset of patients, and multiple daily dosing. Consequently, there is a need for novel therapies to treat $\mathrm{nOH}$. This is where the story of norepinephrine transporter (NET) inhibition in autonomic failure begins.

Blood pressure effects from NET inhibition result from a balance between centrally mediated alpha- 2 effects and a peripheral vasopressor action. Whereas in healthy subjects use of NET inhibitors results in little or no change in blood pressure, in patients with efferent baroreflex failure the peripheral effect prevails [11]. Shibao and colleagues

Guillaume Lamotte

guillaumelamotte14@gmail.com

1 Department of Neurology, University of Utah, Salt Lake City, UT, USA

2 Department of Neurology, Dysautonomia Center, NYU Langone Medical Center, New York, NY, USA first showed that atomoxetine, a NET inhibitor commonly used for the treatment of attention deficit and hyperactivity disorder (ADHD), induced a hypertensive response in a small group of patients with central autonomic failure, even at low doses, with an efficacy comparable to midodrine. The pressor response to atomoxetine seems to be positively correlated with plasma norepinephrine levels $[10,12,13]$.

Enter ampreloxetine, a novel long-acting inhibitor of the cell membrane NET. In this issue of Clinical Autonomic Research, Kaufmann and colleagues report the safety and efficacy of ampreloxetine to treat $\mathrm{nOH}$, in a small phase 2 clinical trial [5]. This phase 2 multicenter study of ampreloxetine included three different parts [5]. In Part A, participants received a placebo on day 1 and an ascending dose of ampreloxetine (1-20 mg) on days $2-5$. The primary endpoint was the change in seated systolic blood pressure (SBP) 6-8 $\mathrm{h}$ post-ampreloxetine. Responders were identified by an increase of $\geq 10 \mathrm{mmHg}$ in seated SBP with ampreloxetine versus placebo. In Part B, responders were randomized (1:1) to ampreloxetine, at the dose of 1.5 times their highest effective tolerated dose during Part A, or placebo for one day and the same primary endpoint was used. Part B enrollment was terminated early after the inclusion of only 10 patients due to subjective improvement in the ampreloxetine group that lead to an amendment of the protocol. Part $\mathrm{C}$ was an openlabel extension phase where participants received ampreloxetine (starting dose of $50 \%$ of the highest effective tolerated dose in Part A with subsequent increase up to $20 \mathrm{mg}$ once daily) for 20 weeks followed by 4 weeks of withdrawal. The primary endpoint for Part $\mathrm{C}$ was the change from baseline in the first question of the Orthostatic Hypotension Symptom Assessment (OHSA \#1) scores at week 4. OHSA \#1 assesses subjective dizziness, lightheadedness, feeling faint, or feeling like blacking out. The score ranges from 0 to 10 , with 10 being the most severe. Ampreloxetine was overall well tolerated. During Part B, seated SBP was significantly 
higher with ampreloxetine compared to placebo after $4 \mathrm{~h}$ with no significant difference at 7,9 , and $12 \mathrm{~h}$, in spite of the medication's extended half-life. The change in standing SBP was not different between the two groups either. During Part $C$ there was a subjective clinical improvement with ampreloxetine in symptomatic participants (OHSA\#1 $>4$ at baseline) with a sustained effect for 20 weeks and a return to baseline levels of symptoms after withdrawal. An increase in standing SBP was observed throughout Part C, even during withdrawal [5].

The study has some limitations, such as its relatively small sample size, particularly in Part B, and the lack of a control group in Part $\mathrm{C}$. The primary endpoint was not met for Part B because the change in seated SBP 6-8 h after drug administration was not different between the treatment and placebo groups, which may indicate a shorter duration of action of ampreloxetine than previously thought. Importantly, the change in standing SBP, which may be considered a more relevant endpoint of treatment efficacy, was not different between ampreloxetine and placebo.

In spite of these limitations, the study results were considered very promising and Theravance Biopharma, the company sponsoring the development of ampreloxetine, went on to implement a large randomized, double-blind, placebo-controlled phase 3 clinical trial program to confirm the safety and efficacy of ampreloxetine for $\mathrm{nOH}$. The phase 3 trial started on 24 January 2019 and recruitment finished on 17 June 2021. A recent press release from the company, however, announced the failure of the phase 3 study due to a lack of efficacy [1]. Although not yet publicly available, the disappointing outcome of the ampreloxetine phase 3 study raises several questions and considerations on the pitfalls in the interpretation of phase 2 trials, and offers an opportunity to avoid these in future clinical trials for $\mathrm{nOH}$.

The key question is: did the phase 2 study provide the necessary criteria for moving ampreloxetine to a phase 3 clinical trial program in patients with $\mathrm{nOH}$ ? Examples of potential reasons for phase 3 failure include an incomplete understanding of target disease biology, a lumping of different diseases with different pathophysiological mechanisms, inadequate dose finding in the phase 2 study, overoptimistic assumptions of the phase 2 results, missing data, attrition bias, and the use of a biomarker in phase 2 that did not accurately predict the phase 3 outcome [8].

What primary endpoint should be used to investigate novel therapies for $\mathrm{nOH}$ ? Questionnaires describe the functional status of patients and they are often used in clinical trials, however, relying on a single item may have underestimated the benefit of ampreloxetine on other domains such as standing, walking, cognition, and other aspects of quality of life. Patient-reported questionnaires are inherently subjective, which leaves a place for personal interpretation that can lead to increase the variability of the data. Furthermore, symptoms often vary from day to day and fluctuate throughout the day, which makes challenging for the clinician and the patient to assess overall improvement. Composite scores such as the composite overall OHQ score have been used as primary end-points in successful phase 3 clinical trials that led to drug approval [8]. Would standing blood pressure be a better alternative primary endpoint? Measurement of blood pressure has the advantage of being objective, however, this endpoint may lack clinical relevance because symptoms are not always related to blood pressure in patients with $\mathrm{nOH}$. Conversely, a similar rise in blood pressure may be effective in mildly affected patients, whereas it may not be enough to provide sufficient relief in patients with a more severe orthostatic drop in blood pressure. In the phase 2 ampreloxetine trial, symptoms of orthostatic intolerance returned to pretreatment levels after 4 weeks of withdrawal while changes in standing SBP remained stable [5]. This suggests that ampreloxetine may have a beneficial effect that is independent of blood pressure changes. The placebo response should also be carefully evaluated and can be impressive in patients with $\mathrm{nOH}$. Moreover, mood disorders may not have been accounted for during this study, even though they can negatively affect how patients perceive and report their symptoms [6]. Other factors to consider include how much blinding is considered in the absence of randomization: during part A, patients received placebo followed by increasing doses of ampreloxetine in a fixed way. Moreover, the product was administered dissolved in apple juice. Changes in flavor during subsequent days may have reduced the effect of blinding, as taste-matching can be a challenge in placebo design, and taste itself influences in the placebo response [14]. Finally, there was no mention of non-pharmacological measures used by patients to treat $\mathrm{nOH}$.

Although efferent baroreflex failure is characteristic of the synucleinopathies, the sites of the lesions vary. Postganglionic sympathetic nerves are more affected in Lewy body disorders (pure autonomic failure and Parkinson disease) compared with multiple system atrophy, which has primarily a preganglionic (i.e., central) autonomic involvement [7]. Ampreloxetine was expected to produce a larger pressor effect in patients with intact peripheral noradrenergic innervation than in patients with peripheral noradrenergic denervation. In support of this assumption, Shibao and colleagues reported that plasma norepinephrine level was modestly associated with the pressor response of atomoxetine in patients with nOH [13]. Subgroup analysis of the results of the phase 3 study should consider the nature of the pre-and post-ganglionic lesions and plasma norepinephrine levels.

Unfortunately, failure of phase 3 trials despite promising phase 2 trials is not rare. The effects of phase 3 failures are significant for patients, caregivers, clinicians, researchers, and pharmaceutical companies. The use of a larger sample size in phase 2 trials seems to be the simplest and most 
intuitive way to reduce phase 3 failures [2]. Nevertheless, a lot can be learned from failed clinical trials, and a careful analysis of the phase 3 trial might perhaps identify a small subset of patients who would benefit from ampreloxetine. Understanding the pathophysiology and neural pathways involved in $\mathrm{nOH}$ is crucial to developing effective treatments and future studies should consider the differences between Lewy body disorders and multiple system atrophy. Careful consideration of the study design, primary and secondary endpoint selection, patient recruitment, and retention strategies is key when planning a clinical trial for $\mathrm{nOH}$. Adaptive designs with larger sample sizes and the use of biometric monitoring devices to accurately and objectively reflect the disease process and status of patients during their daily lives should also be considered in future trials.

All in all, the phase 2 trial for ampreloxetine promised a novel drug that would have provided long-acting relief for patients with $\mathrm{nOH}$. As this chapter reaches its end, pending the final publications of the phase 3 trial that disappointingly failed to meet its endpoints, we impatiently await the results of another phase 2 trial that is investigating the effect of atomoxetine in the symptomatic management of nOH (ClinicalTrials.gov: NCT02796209). The story for norepinephrine reuptake inhibition for $\mathrm{nOH}$ has not ended yet, and we hope to have evidence to support the addition of a much-needed new drug to our small armamentarium.

Funding None.

\section{Declarations}

Conflict of interest Dr. Millar Vernetti was a site principal investigator of the Theravance-sponsored clinical trials; he received research funding from Theravance Biopharma. Dr. Lamotte has no conflicts.

\section{References}

1. Biopharma (Theravance Biopharma) (2021) Press release: Thervance Biopharma Inc. Announces Top-line Results from a Phase 3 Study of Ampreloxetine in Patients with Symptomatic Neurogenic Orthostatic Hypotension. https://www.prnew swire.com/news-releases/theravance-biopharma-inc-annou nces-top-line-results-from-a-phase-3-study-of-ampreloxetine-inpatients-with-symptomatic-neurogenic-orthostatic-hypotension301377200.html

2. De Martini D (2020) Empowering phase 2 clinical trials to reduce phase 3 failures. Pharm Stat 19:178-186

3. De Pablo-Fernandez E, Tur C, Revesz T, Lees AJ, Holton JL, Warner TT (2017) Association of autonomic dysfunction with disease progression and survival in Parkinson disease. JAMA Neurol 74:970-976

4. Fanciulli A, Campese N, Goebel G, Ndayisaba JP, Eschlboeck S, Kaindlstorfer C, Raccagni C, Granata R, Bonuccelli U, Ceravolo R, Seppi K, Poewe W, Wenning GK (2020) Association of transient orthostatic hypotension with falls and syncope in patients with Parkinson disease. Neurology 95:e2854-e2865

5. Kaufmann H, Vickery R, Wang W, Kanodia J, Shibao CA, Norcliffe-Kaufmann L, Haumann B, Biaggioni I (2021) Safety and efficacy of ampreloxetine in symptomatic neurogenic orthostatic hypotension: a phase 2 trial. Clin Auton Res (online ahead of print)

6. Martinez J, Palma JA, Norcliffe-Kaufmann L, Garakani A, Kaufmann H (2020) Impact of depressive symptoms on self-perceived severity of autonomic dysfunction in multiple system atrophy: relevance for patient-reported outcomes in clinical trials. Clin Auton Res 30:215-221

7. Palma J-A, Mano T (2018) Central or peripheral autonomic dysfunction in Parkinson disease. Does it matter? Neurology 90:1045-1046

8. Palma JA, Kaufmann H (2020) Clinical trials for neurogenic orthostatic hypotension: a comprehensive review of endpoints, pitfalls, and challenges. Semin Neurol 40:523-539

9. Park JW, Okamoto LE, Shibao CA, Biaggioni I (2020) Pharmacologic treatment of orthostatic hypotension. Auton Neurosci 229:102721

10. Ramirez CE, Okamoto LE, Arnold AC, Gamboa A, Diedrich A, Choi L, Raj SR, Robertson D, Biaggioni I, Shibao CA (2014) Efficacy of atomoxetine versus midodrine for the treatment of orthostatic hypotension in autonomic failure. Hypertension 64:1235-1240

11. Schroeder C, Jordan J (2012) Norepinephrine transporter function and human cardiovascular disease. Am J Physiol 303:H1273-H1282

12. Shibao C, Raj SR, Gamboa A, Diedrich A, Choi L, Black BK, Robertson D, Biaggioni I (2007) Norepinephrine transporter blockade with atomoxetine induces hypertension in patients with impaired autonomic function. Hypertension 50:47-53

13. Shibao CA, Palma JA, Celedonio JE, Martinez J, Kaufmann H, Biaggioni I (2021) Predictors of the pressor response to the norepinephrine transporter inhibitor, atomoxetine, in neurogenic orthostatic hypotension. Hypertension 78:525-531

14. Wan M, Orlu-Gul M, Legay H, Tuleu C (2013) Blinding in pharmacological trials: the devil is in the details. Arch Dis Child 98:656-659 